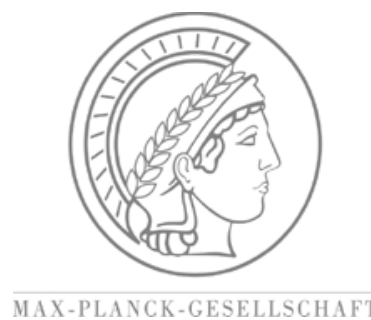

\# 1109

Social Dilemmas, Time Preferences and Technology Adoption in a Commons Problem

Reinoud Joosten

The Papers on Economics and Evolution are edited by the Evolutionary Economics Group, MPI Jena. For editorial correspondence, please contact: evopapers@econ.mpg.de 


\title{
Social Dilemmas, Time Preferences and Technology Adoption in a Commons Problem
}

\author{
Reinoud Joosten*
}

June 14, 2011

\begin{abstract}
Agents interacting on a body of water choose between technologies to catch fish. One is harmless to the resource, as it allows full recovery; the other yields high immediate catches, but low(er) future catches.

Strategic interaction in one 'objective' resource game may induce several 'subjective' games in the class of social dilemmas. Which unique 'subjective' game is actually played depends crucially on how the agents discount their future payoffs. We examine equilibrium behavior and its consequences on sustainability of the common-pool resource system under exponential and hyperbolic discounting.

A sufficient degree of patience on behalf of the agents may lead to equilibrium behavior averting exhaustion of the resource, though full restraint (both agents choosing the ecologically or environmentally sound technology) is not necessarily achieved. Furthermore, if the degree of patience between agents is sufficiently dissimilar, the more patient is exploited by the less patient one in equilibrium.

We demonstrate the generalizability of our approach developed throughout the paper. We provide recommendations to reduce the enormous complexity surrounding the general cases.
\end{abstract}

JEL codes: C72, C73, Q22, Q57.

Keywords: stochastic renewable resource games, hyperbolic \& exponential discounting, social dilemmas, sustainability.

\section{Introduction}

The central theme of this paper is the effect of time preferences in a commons problem on the adoption of a technology. To be more specific, the resource problem investigated is that of a Small Fish War (Joosten [2007a,b,c]) to be characterized as follows. ${ }^{1}$ Several agents possess the fishing rights to a

${ }^{*}$ I thank Christian Cordes, Sebastiaan Morssinkhof and Berend Roorda for comments. Address: School of Management \& Governance, University of Twente, POB 217, 7500 AE Enschede, The Netherlands. Email: r.a.m.g.joosten@utwente.nl

${ }^{1}$ A word play on Levhari \& Mirman [1980] who show that strategic interaction in a fishery may induce a 'tragedy of the commons' (Hardin [1968]). 
body of water, and they have essentially two options, to fish with or without restraint. 'Restraint' in practice may take various forms, e.g., regarding catching seasons, quantities caught, catching methods, technologies, e.g., boats, nets, allowed in catching. Unrestrained fishing yields a higher immediate catch, but it may lead to a decreasing fish stock and hence, decreasing future catches in the long run. Restrained fishing by both agents is assumed to be sustainable.

In a 'standard' Small Fish War agents maximize their average catches over an infinite time-horizon. In such a setting, a 'tragedy of the commons' does not seem inevitable, as Pareto-efficient outcomes can be sustained by subgame perfect equilibria. In a wide range of the parameter space of the model, the more the catches deteriorate due to over-fishing, the greater the gap between Pareto-efficient outcomes and the 'never restraint' outcome, but the smaller the gap between the former and the 'perfect restraint' outcome.

The choice of a technology by two agents takes place at the start of the game and can not be undone for a sufficiently long period of time. For the sake of simplicity, each agent can choose between two alternative technologies and has to consider the following. One technology yields a higher immediate payoff but damages the resource; the alternative yields a lower immediate payoff but allows the resource to recover completely. We assume that the use of the harmful technology by both agents damages the fish stock sufficiently such that long-term catches deteriorate to a level below the full-restraint level.

By focussing analysis on technology choice between an environmentally neutral and a detrimental one, we can show links to and make comparisons with contributions in the social dilemma literature, cf., e.g., Komorita \& Parks [1994], Heckathorn [1996], Marwell \& Oliver [1993]. The resource game is to be associated primarily with a social trap ${ }^{3}$, see e.g., Platt [1973], Cross \& Guyer [1980] and the closely related 'tragedy of the commons' cf., e.g., Hardin [1968], Messick et al. [1983], Messick \& Brewer [1983]).

The modelling and analysis of social traps involves time in a non trivial manner. First, current (past) actions have an influence on future (present) stage payoffs. To be a little bit more specific, a social trap is a situation in which a certain action always induces a higher immediate stage payoff regardless of what the other agents do, but the continued playing of this dominant action in the stage games leads to considerably lower future stage payoffs on all actions. Furthermore, it matters how much agents care about the future. Very 'myopic' agents only care about the present payoffs, hence they are very likely to choose the action yielding the immediate advantage.

As in more traditional Small Fish Wars (e.g., Joosten [2007a,b,c]) a

\footnotetext{
${ }^{2}$ Term is due to Hardin [1968], yet the underlying problem it was already recognized in antiquity. An earlier classic, related to the present context, is Gordon [1954].

${ }^{3}$ Called a 'take some' game by Hamburger [1973] who also introduces 'give some' games. A well-known example of latter is a public good game.
} 
continued use of the ecologically damaging technology by both agents, the long-term catches deteriorate below the level of the catches under perfect restraint. Furthermore, we specify what occurs in the long run if one agent chooses restraint and the other one chooses the environmentally damaging technology. This covers the first non-trivial influence of time as mentioned, all aspects here are 'objective', in the sense that one can actually measure the effects of the agents' choices.

The next point of focus is the way the agents care about the future, i.e., evaluate their streams of future catches at the moment of the technology adoption decision. First, we examine the game under the assumption that the players use exponential discounting to evaluate their infinite streams of stage payoffs. For this evaluation criterion every unit of payoffs $K$ periods removed in the future from any point $t$ in the future receives weight $\beta^{K-1}$, $\beta \in[0,1)$, times the weight of the same unit of payoffs receives in period $t$ (cf., e.g., Samuelson [1937]). Following standard interpretations, e.g., Koopmans [1960], we say that an agent is more patient for higher values of $\beta$. Second, we examine the game under the assumption that the players use hyperbolic discounting (e.g., Phelps \& Pollack [1968]), i.e., every unit of payoffs $t$ periods removed in the future is evaluated at $\frac{1}{1+D t}, D \geq 0$, times an immediate payoff of one unit. Here, an agent is said to be more patient for smaller $D$, as for smaller values future payoffs receive more weight.

For expository purposes we examine a particular Small Fish War extensively for the possible effects of discounting under different degrees of patience distinguishing four different ranges. Very impatient agents play a game in which it is beneficial for both to choose the ecologically harmful technology as this strategy is the dominant action and the associated rewards are the next-to-highest available. Less impatient agents are involved in a Prisoners' Dilemma, with the usual problem that the Nash equilibrium, i.e., mutual no-restraint, yields a lower reward than the ones associated with full restraint. Both situations, i.e., a pair of very impatient agents and a pair of impatient agents, induce games in which it is rational to exploit the resource ruthlessly. Moderately patient agents are involved in a so-called Chicken Game which has two asymmetric pure Nash equilibria. In either equilibrium one agent chooses restraint, the other chooses no restraint. The long term fish stock will be considerably higher than if both choose norestraint, yet lower than if both were to choose restraint. Patient agents induce a so-called Privileged Game, i.e., both agents choose restraint and this equilibrium gives to each player the most preferred reward of all rewards possible, meanwhile the resource remains at full capacity.

In the preceding paragraph, we presented the results for the four different ranges of patience, assuming that both agents belong to the same category. We have also performed the same analysis for players belonging to different regions of patience. The total number of games is six, given the four possible ranges of patience. In all but one game, the more patient agent receives 
his second worst reward possible, the more impatient one, the best reward possible. For the resource, the outcome is moderate as in all resulting games, the more patient agent chooses restraint and the impatient one chooses norestraint. In the remaining game, the more patient one still receives his second worst reward, yet the more impatient one the second-best reward. The resource is fully exploited and the long term fish stock will be at their technically feasible minimum level.

As a sensitivity analysis we examine the robustness of results with respect to changes in the parameters concerning the resource stock dynamics. Next to an affirmative answer to this robustness question, interesting stylized facts have been found. Summarizing them, it seems that the more reactive the system is to overfishing at the fish stock maximum level, the 'earlier' the agents come to their senses and equilibrium behavior leads to more sustainable outcomes. By earlier, we mean that the favorable changes of the discounted game occur at lower levels of $\beta$ or higher levels of $D$. Assuming the agents' time preferences to be drawn from a given distribution, chances for the resource improve. An explanation is that the more near future payoffs are similar to the ones obtained at present, the more the discounted games resemble the present stage game.

To give the reader an idea about the generalizability of our endeavours, we examine a Stag Hunt in full. We investigate the set of possible discounted games which may arise from a Stag Hunt in a Small Fish War, and obtain a small universe of games. For sufficiently symmetric time preferences, this universe consists of eight games, only one of which is a Prisoners' Dilemma. The eight games are connected by non-degenerate, symmetric and admissible transitions in structure. We call the original Stag Hunt the starting game and one of the other games the end game. From the starting game, ten paths emanate to end games and it is situation-dependent which path is relevant given the dynamics of the resource. The more interesting paths contain several transitions, the largest number of them being four, i.e., one more than in the example studied. The largest number of paths connecting the same starting and ending games is three.

This universe arising from the Stag Hunt has acceptable complexity, yet dropping for instance symmetry makes the universe expand enormously. For this situation a complete overview must be regarded as unworkable. We have therefore two recommendations for practical purposes. To focus on one particular resource game and examine it in full as we did in our example for expository purposes. The second recommendation is to look only at the changes relevant in a game-theoretical sense, as these total at most two, and perhaps more importantly, they are the changes relevant to the question whether the resource will be sustainable or not in equilibrium. The first neglects other games possibly arising from the same setting, the second one neglects information about interesting social dilemma.

Next, we recall the small Fish War. Section 3 presents several social 
dilemmas to be obtained in our model of technology choice in a Small Fish War by both exponential and hyperbolic discounting. We perform a sensitivity analysis in Section 4 . Section 5 extends the analysis shown by means of example in Section 3. Section 5 concludes.

\section{Fishing in a vulnerable environment}

The Small Fish War is played by players $A$ and $B$ at discrete moments in time called stages. Each player has two actions and each stage each player independently and simultaneously chooses an action. We denote the action set of player $A(B)$ by $J^{A}=\{0,1\}\left(=J^{B}\right)$ and $J \equiv J^{A} \times J^{B}$. Action 1 for either player denotes the action without or with very little restraint, e.g., catching with fine-mazed net or catching a high quantity. Action 0 denotes the action where there exists some restriction, i.e., catching with wide-mazed nets or catching a low quantity. The payoffs at stage $t^{\prime} \in \mathbb{N}$ of the play depend on the choices of the players at that stage, and on the relative frequencies with which all actions were actually chosen until then.

Let $h_{t^{\prime}}^{A}=\left(j_{1}^{A}, \ldots, j_{t^{\prime}-1}^{A}\right)$ be the sequence of actions chosen by player $A$ until stage $t^{\prime} \geq 2$, let $h_{t^{\prime}}^{B}=\left(j_{1}^{B}, \ldots, j_{t^{\prime}-1}^{B}\right)$ be defined similarly and let $q \geq 0$. Then, define the rate of overfishing $\rho_{t}$ recursively for $t \leq t^{\prime}$ by

$$
\rho_{1}=\rho \in[0,1] \text {, and } \rho_{t}=\frac{q+t-1}{q+t} \rho_{t-1}+\frac{1}{q+t}\left(\frac{j_{t-1}^{A}+j_{t-1}^{B}}{2}\right) \text {. }
$$

Taking $q \gg 0$ serves to moderate 'early' effects. Note that for the long run rate of overfishing the choice of numbers $\rho$ and $q$ is irrelevant.

At stage $t \in \mathbb{N}$, the normalized fish stock $\mu_{t}$ is given by

$$
\mu_{t} \equiv 1+(1-\underline{m})\left[\frac{n_{2}}{n_{1}-n_{2}} \rho_{t}^{n_{1}}-\frac{n_{1}}{n_{1}-n_{2}} \rho_{t}^{n_{2}}\right],
$$

where $\underline{m} \in[0,1]$ represents the minimal stock due to overexploitation by the agents, and $n_{1}>n_{2}>1$. So, Eq. (2) determines how the fish stock evolves due to fishing without restraint.

At each stage a bi-matrix game is played, and the choices of the players at that stage determine their stage payoffs. Let

$$
A=B^{\top}=\left[\begin{array}{ll}
a & b \\
c & d
\end{array}\right]
$$

Then, for given $\mu_{t} \in[0,1]$ at stage $t \in \mathbb{N}$, the stage payoffs are given by

$$
\left[\begin{array}{cc}
a \mu_{t}, a \mu_{t} & b \mu_{t}, c \mu_{t} \\
c \mu_{t}, b \mu_{t} & d \mu_{t}, d \mu_{t}
\end{array}\right]
$$


Here, $\mu_{t}$ may be interpreted as a measure for the present fish stock; if player $A$ chooses action 0 and $B$ chooses action $1, A$ 's stage payoff is $b \mu_{t}$ and $B$ 's is $c \mu_{t}$. We assume that fishing without restraint yields a higher catch in any current stage than fishing with restraint, hence $a<c, b<d$. We assume that two-sided catching without restraint yields higher immediate payoffs than two-sided catching with restraint, i.e., $a<d$. Finally, we assume that the player catching without restraint is better off than his opponent if the latter catches with restraint, hence $b<c$. The unique stage-game equilibrium is the strategy pair in which both players use action 1 .

Observe that for $\underline{m}=1$, we have a standard repeated game. The part between the brackets determines the sensitivity of the fish stock to overexploitation. For increasing $n_{1}$, the deterioration of the fish stock near its maximum, is less and less noticeable; as a consequence the descent 'later on' must be steeper, the collapse of the fish stock is very rapid indeed. Below, (2) is visualized for $\underline{m}=0.1, n_{2}=n_{1}-1$, and different values of $n_{1}$; the greater $n_{1}$, the higher the corresponding curve. For the six lower curves $n_{1}$ lies between 2.2 and 5 ; the highest curve has $n_{1}=100$. For the latter value of $n_{1}$, noticeable effects on the fish stock are to be found when e.g., both agents fish without restraint for approximately $90 \%$ of the time.

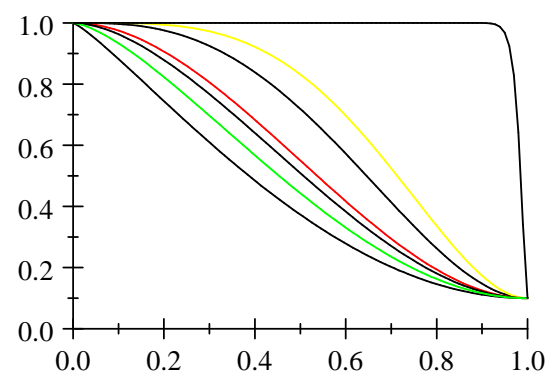

If both agents never show restraint, then their associated long run stage payoffs are $d \underline{m}$; under perfect restraint, their long run stage payoffs equal $a$. For large $n_{1}, n_{2}$ unrestrained fishing can go on for quite a while without having a noticeable effect on the environment. Conversely, for $n_{1} \approx n_{2}$ or fixed $n_{1}$ and $n_{2} \downarrow 1$, the environment is extremely sensitive.

The setting is supposed to be similar to a social trap. For the parameters of the model we therefore arrive at yet another restriction. In words, the continued use of the dominant action by both agents in the stage game, namely to catch without restraint, leads to a situation in which the fish stock deteriorates to such a level that the use of this action yields a pair of immediate payoffs which are lower than the alternative would have provided the latter would have been used at all preceding stage games. A restriction which takes care of this aspect is $a>d \underline{m}$.

The agents are assumed to choose one of two catching technologies. One 
technology is harmless to the fish stock and the associated immediate catches can be sustained forever; the other one is harmful to the fish stock but yields (in every situation) a higher immediate catch than the alternative, in the long run the catches might deteriorate significantly. As an example of a catching technology one may think of a type of a boat, an engine, or some fishing gear. Crucial is that it is assumed that the technology chosen can not be replaced in a reasonable amount of time, or that it is so expensive that it is not feasible to possess the alternative simultaneously and the associated investments are sunk and can not be recovered.

As mentioned in the introduction this assumption of a once and for all technology choice simplifies the analysis. We only need to consider so-called 'simple pure strategies', i.e., $\pi(i)=(i, i, i, \ldots)$ and $\sigma(j)=(j, j, j, \ldots)$ for $i, j \in\{0,1\}$. Randomization over these strategies is allowed in the sense that the adoption of a simple pure strategy might be thought of as being preceded by randomization by one agent or both.

\section{Social dilemmas and discounting}

In the literature on social dilemmas (cf., e.g., Komorita \& Parks [1994], Heckathorn [1996]) bi-matrix games of the following types

$$
\left[\begin{array}{ll}
\varphi, \varphi & \chi, \psi \\
\psi, \chi & \omega, \omega
\end{array}\right]
$$

are frequently studied where we may have:

- $\psi>\varphi, \omega>\chi$, and $\varphi>\omega$, i.e., the P(risoners') $\mathrm{D}$ (ilemma);

- $\psi>\varphi, \omega<\chi$, and $\varphi>\omega$, i.e., the $\mathrm{CH}($ icken Game);

- $\psi<\varphi, \omega<\chi$, and $\varphi>\omega$, i.e., the $\mathrm{P}$ (rivileged) $\mathrm{G}$ (ame).

- $\chi<\omega<\psi<\varphi$, i.e., the $\mathrm{S}(\operatorname{tag}) \mathrm{H}($ unt $)$.

In the social dilemmas literature, the Prisoners' Dilemma takes a very prominent role. In the 'PD-terminology' the left (top) action denotes 'to cooperate' and the alternative 'to defect'. The payoffs have names of their own as well. In the $\mathrm{PD} \psi$ is called the ' $\mathrm{T}$ (emptation)', $\varphi$ is called ' $\mathrm{C}$ (ooperation)', $\omega$ is ' $\mathrm{P}$ (unishment)' and $\chi$ is ' $\mathrm{S}$ (ucker reward)'. Sometimes the (abbreviations of the) names of the payoffs and actions are transferred to other social dilemmas, or even to games having even less in common with the PD.

The salient feature of a PD is that it possesses a unique Nash equilibrium in dominant pure strategies, but there exists another pair of pure strategies which Pareto-dominates the Nash equilibrium, yet is not an equilibrium itself. A Chicken Game has two pure Nash equilibria in which both players 
anti-coordinate on their pure actions. An SH has two pure Nash equilibria in which both players coordinate their pure actions, and the Pareto-dominant Nash equilibrium is riskier than the Pareto-inferior one. To help the discussion, let $\left(s_{D}, s_{D}\right)$ be the Pareto-dominant Nash equilibrium and $\left(s_{I}, s_{I}\right)$ be the Pareto-inferior one. Suppose that one agent plays $s_{D}$ expecting that the other plays the strategy $s_{D}$. If the first player is mistaken, he receives the lowest payoff possible. Choosing $s_{I}$ instead guarantees a higher amount regardless of the opponent's action.

The structure relevant for a game-theoretical analysis of these games depends on $\varphi-\psi$ and $\chi-\omega$. What makes it a social dilemma however, is that $\varphi>\omega$, i.e., if all agents cooperate, the associated payoffs are higher than if all defect (cf., e.g., Schelling [1978], Liebrand [1983], Dawes [1980]).

The Prisoners' Dilemma is due to Merrill Flood and Melvin Dresher and received its name and famous interpretative anecdote by Albert Tucker (cf, e.g., Campbell [1985, p.3], Poundstone [1992]). The name Chicken Game appeared first in Kahn [1965], an alternative term for such a game is Snowdrift (cf., e.g., Skyrms [1996], Sigmund [2010]). The name Privileged Game is due to Heckathorn [1996] inspired by Olson [1965]. The Stag Hunt can be traced back to at least Rousseau [1755], though Skyrms [2001] quotes Hobbes [1651] and Hume [1739] as earlier examples of the same kind. A Stag Hunt is sometimes called an Assurance Game (Sen [1967], Heckathorn [1996]) or a Trust Game (Liebrand [1983]).

In the following two subsections we show that one and the same resource may induce different games associated with different levels of patience of the agents. Please keep in mind that the objective situation is taken predetermined, only the perception of the agents regarding to which game they are playing changes in response to their patience. In this respect there is some analogy to an approach in Skyrms [2001], where a Prisoners' Dilemma is repeatedly played and the infinite repetition of the same one-shot PD is evaluated as a Stag Hunt by adding a punishment strategy for each player.

Though intellectually indebted to contributions from e.g., Heckathorn [1996], Dudley \& Witt [2004], Dudley [2000], where also several social dilemmas arise from one meta-game, it should be noted that these different games are to be regarded as stage games, arising through time as a result of choices made in the past. In our paper, we have one objective resource game, admittedly consisting of an array of stage games resulting from actual historic choices. However, we have also a multitude of subjective games at the decision point caused by the manner in which agents evaluate future payoffs.

Here the top (left) action is to choose the strategy which allows full recovery of the fish stock, whereas the alternative is to choose the one which causes the fish stocks to decrease from maximum level. 


\subsection{Exponential discounting}

In economics, exponential discounting is a widespread and fairly standard method to compare streams of payoffs distributed over several distinct stages. Contrastingly, there is also a strand of the literature considering long-term average payoffs which is deemed particularly useful for environmental considerations (see e.g., Schelling [1995], Heal [1998], Weitzman [1998, 2001]). Our earlier Small Fish Wars used the so-called limiting average reward criterion for analysis. Though theoretically possible, cf., e.g., Fudenberg \& Maskin [1986], it is very cumbersome to obtain tangible results similar to the ones presented in e.g., Joosten et al. [2003], Joosten [2007a,b,c] for the unrestricted case. ${ }^{4}$ However, due to our assumption that each agent must choose one technology for the entire game, the repeated game can be reduced to a one-shot game by discounting the stream of future stage payoffs.

Given strategy pair $(\pi, \sigma)$ and $\beta \in[0,1)$, player $k$ 's $\beta$-discounted rewards, $k=A, B$, are given by $\gamma_{\beta}^{k}(\pi, \sigma)=(1-\beta) \sum_{t=1}^{\infty} \beta^{t-1} R_{t}^{k}(\pi, \sigma)$. The part before the summation sign is a normalization which guarantees that a constant stream of payoffs of say $a$ is evaluated as $a$. A low (high) $\beta$ means that the agent is motivated by short (long) term considerations in evaluating the stream of stage payoffs. Less formally stated, a high (low) $\beta$ may be associated with (im)patient agents (cf., e.g., Koopmans [1960]).

Next, we consider the family of matrices depending on the discount factor

$$
\left[\begin{array}{ll}
\varphi_{\beta}, \varphi_{\beta} & \chi_{\beta}, \psi_{\beta} \\
\psi_{\beta}, \chi_{\beta} & \omega_{\beta}, \omega_{\beta}
\end{array}\right]
$$

where we define $\varphi_{\beta}=\gamma_{\beta}^{1}(\pi(0), \sigma(0))=\gamma_{\beta}^{2}(\pi(0), \sigma(0)), \psi_{\beta}=\gamma_{\beta}^{1}(\pi(1), \sigma(0))$ $=\gamma_{\beta}^{2}(\pi(0), \sigma(1)), \chi_{\beta}=\gamma_{\beta}^{1}(\pi(0), \sigma(1))=\gamma_{\beta}^{2}(\pi(1), \sigma(0))$, and finally $\omega_{\beta}=$ $\gamma_{\beta}^{1}(\pi(1), \sigma(1))=\gamma_{\beta}^{2}(\pi(1), \sigma(1))$.

Below, we visualize effects $\beta$ on the structure of the game. ${ }^{5}$

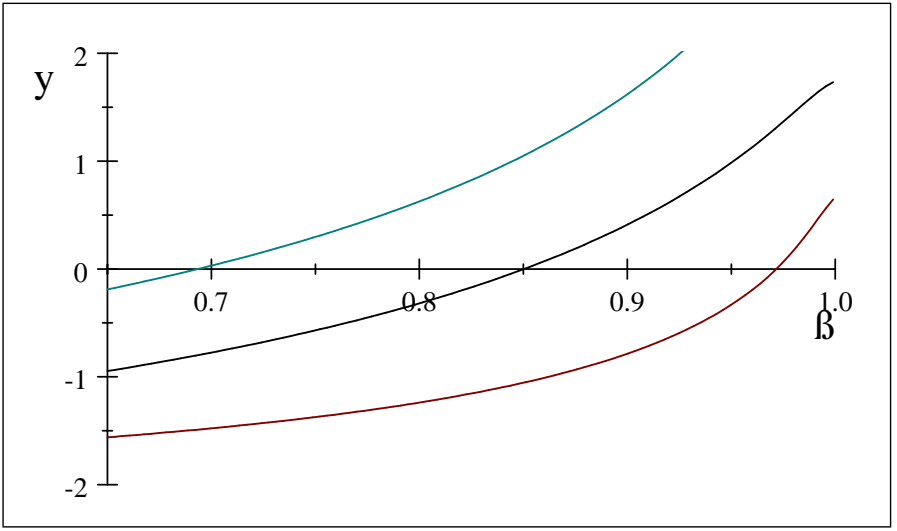

\footnotetext{
${ }^{4}$ Efficient algorithms to obtain large sets of feasible $\beta$-discounted rewards are lacking.

${ }^{5}$ To save computing time the infinite sum was cut off after $t=250 ; \underline{m}=0, n_{1}=3$, $n_{2}=2, q=5, a=4, b=\frac{7}{2}, c=6, d=\frac{11}{2}$.
} 
The blue-green curve represents $\varphi_{\beta}-\omega_{\beta}$; let us refer to the value where it intersects the $\beta$-axis as $\beta_{\text {left }}$. The black curve represents $\chi_{\beta}-\omega_{\beta}$; it intersects the $\beta$-axis at $\beta_{\text {middle }}$. The red curve represents $\varphi_{\beta}-\psi_{\beta}$; it intersects the $\beta$ axis at $\beta_{\text {right }}$. For $\beta>\beta_{\text {left }}$ we have a social dilemma., i.e., the socially defective choice is Pareto dominated by the socially cooperative one. For $\beta>\beta_{\text {middle }}$ bottom-right can never be associated with an equilibrium. For $\beta>\beta_{\text {right }}$ top-left is turned into an equilibrium. We now can give the following overview regarding the time preferences using ordinal ranking as in Guyer \& Rapoport [1966], where 4 denotes the highest utility, 1 lowest.

- Very impatient $\left(\beta<\beta_{\text {left }}\right)$ : The corresponding matrix is

$$
M_{V V}=\left[\begin{array}{ll}
2,2 & 1,4 \\
4,1 & 3,3
\end{array}\right] .
$$

Each player has a dominant action, namely to defect; the socially defective choice induces an equilibrium which Pareto-dominates the socially cooperative one. Hence, the game is not even a social dilemma. The fish stock will be depleted maximally, i.e., $\lim _{t \rightarrow \infty} \mu_{t}=\underline{m}$.

- Impatient $\left(\beta_{\text {left }}<\beta<\beta_{\text {middle }}\right)$ : The corresponding matrix is

$$
M_{I I}=\left[\begin{array}{ll}
3,3 & 1,4 \\
4,1 & 2,2
\end{array}\right] .
$$

Here, the unique Nash equilibrium is no restraint for both agents. So, there is little hope of reconciling the agents' interests with sustainability of the fish stock in this Prisoners' Dilemma. The fish stock will be depleted, i.e., $\lim _{t \rightarrow \infty} \mu_{t}=\underline{m}$ unless the agents solve the dilemma.

- Moderately patient $\left(\beta_{\text {middle }}<\beta<\beta_{\text {right }}\right)$ : The associated matrix is

$$
M_{M M}=\left[\begin{array}{ll}
3,3 & 2,4 \\
4,2 & 1,1
\end{array}\right] .
$$

In this Chicken Game, in each pure Nash equilibrium one agent always catches with restraint, the other without. The long run fish stock can be computed easily as $\lim _{t \rightarrow \infty} \mu_{t}=\frac{1}{2}$ for the parameters chosen. There is a contradiction between self-interest and sustainability, though less severe than in the preceding case. A complicating factor here is inequality: one agent receives his best and the other his next to worst outcome. Another complication here is coordination: which agent will be the one receiving his best outcome? There is also a mixed Nash equilibrium in every $\mathrm{CH}$ depending crucially on the actual numbers of the four utilities instead of their ordinal ranking. 
- Patient $\left(\beta>\beta_{\text {right }}\right)$ : The corresponding matrix is

$$
M_{P P}=\left[\begin{array}{ll}
4,4 & 2,3 \\
3,2 & 1,1
\end{array}\right] \text {. }
$$

In this Privileged Game, the agents care for high long-term yields. The fish stock remains at maximum level and $\lim _{t \rightarrow \infty} \mu_{t}=1$. So, there is no contradiction between self-interest and sustainability.

The subscripts $P, M, I$ and $V$ indicate that the discounting parameter belongs to the range patient, moderately patient, impatient and very impatient respectively. Here, the first (second) capital denotes the range to which the discount parameter of player 1 (2) belongs.

The analysis can be adapted to incorporate asymmetric time preferences. The actual levels for the discounting parameters are irrelevant, only the ranges matter. If both discount parameters belong to the same range, the overview above applies. The matrices relevant for the asymmetric timepreferences case are the following

$$
\begin{aligned}
M_{P M} & =\left[\begin{array}{cc}
4,3 & 2,4^{*} \\
3,2 & 1,1
\end{array}\right], M_{P I}=\left[\begin{array}{cc}
4,3 & 2,4^{*} \\
3,1 & 1,2
\end{array}\right], M_{P V}=\left[\begin{array}{cc}
4,2 & 2,4^{*} \\
3,1 & 1,3
\end{array}\right], \\
M_{M I} & =\left[\begin{array}{cc}
3,3 & 2,4^{*} \\
4,1 & 1,2
\end{array}\right], M_{M V}=\left[\begin{array}{cc}
3,2 & 2,4^{*} \\
4,1 & 1,3
\end{array}\right], M_{I V}=\left[\begin{array}{cc}
3,2 & 1,4 \\
4,1 & 2,3^{*}
\end{array}\right],
\end{aligned}
$$

Recall that this implies that the row player is the more patient one. In the matrices above, a star denotes equilibrium rewards. $M_{P M}, M_{P I}$ and $M_{M I}$ are also social dilemmas as the socially defective outcome is Pareto dominated by the socially cooperative one. $M_{M I}$ is called a Bully Game in Poundstone [1992] where we suspect a typo occurred, or Called Bluff (Snyder $\&$ Diesing [1977]). The actual game appearing in Poundstone [1992] is $M_{M V}$, a Big Bully Game in Bennet [1998], but this game is not a social dilemma.

Joosten [2005] introduces a Hillel game which has a dominance structure similar to $M_{P M}$ in the following sense. Player A (B) would like his opponent to play the first action because this would yield higher (own) payoffs than the alternative. But in a Hillel game, the Golden Rule ${ }^{6}$ induces a Nash equilibrium which is Pareto optimal. An alternative way of looking at such a game is to alter the utilities of the agents, each agent tries to maximize his opponents payoffs as in Kelley \& Thibaut [1978]. Despite significant similarities with a Hillel game, $M_{P M}$ is different as the Golden Rule does not yield an equilibrium at all.

In all but one matrix the top-right entry is the unique Nash equilibrium reward corresponding to the strategy pair cooperate-defect; in the remaining

\footnotetext{
${ }^{6}$ There are several versions of this rule. The best known ones are: 'Do unto others as you would like them to do unto you' and 'Do not do unto others as you would not like them to do unto you'. The first is attributed to Jesus of Nazareth, the second to Hillel.
} 
one defect-defect is a Nash equilibrium. Hence, the more patient agent obtains the second-worst outcome while the other gets the best one except in one case in which he gets the second best. ${ }^{7}$

\subsection{Hyperbolic discounting}

In behavioral and experimental economics communities hyperbolic discounting introduced by Phelps \& Pollack [1968] is often seen as more appropriate to describe behavior (cf., e.g., Ainslie [1974,1975] Ainslie \& Herrnstein [1981]). ${ }^{8}$ For instance, persistently recurring anomalies in intertemporal choice can be explained better with hyperbolic than with exponential discounting (cf., e.g., Strotz [1952], Rachlin [1970], Rachlin \& Green [1972], Loewenstein \& Prelec [1992], Loewenstein \& Thaler [1998]). If this criticism regarding exponential discounting were found to be valid, then the previous analysis is clearly not sufficient. Therefore, we decided to incorporate the alternative of hyperbolic discounting in a Small Fish War. ${ }^{9}$

For our purposes we normalize the total discounted rewards in such a manner that a stream of constant payoffs amounts to exactly that amount. Hence, we say given strategy pair $(\pi, \sigma)$ and $D \geq 0$, the $D$-discounted rewards of player $k, k=A, B$, are given by

$$
\gamma_{D}^{k}(\pi, \sigma)=\lim _{T \rightarrow \infty}\left(\sum_{t=1}^{T} \frac{1}{1+D(t-1)}\right)^{-1} \sum_{t=1}^{T} \frac{1}{1+D(t-1)} R_{t}^{k}(\pi, \sigma),
$$

where the part $\left(\sum_{t=1}^{T} \frac{1}{1+D(t-1)}\right)^{-1}$ is a normalization guaranteeing that an infinite stream of constant payoffs yield a discounted reward exactly equal to this constant; we take the limit as $\sum_{t=1}^{\infty} \frac{1}{1+D(t-1)}$ is ill-defined. Note that the smaller $D$ the more patient the agent.

Similar to the approach for exponential discounting, we consider the family of matrices depending on the type of discounting

$$
\left[\begin{array}{ll}
\varphi_{D}, \varphi_{D} & \chi_{D}, \psi_{D} \\
\psi_{D}, \chi_{D} & \omega_{D}, \omega_{D}
\end{array}\right]
$$

where for instance $\varphi_{D}=\gamma_{D}^{1}(\pi(0), \sigma(0))=\gamma_{D}^{2}(\pi(0), \sigma(0))$ and the other parameters are computed similarly mutatis mutandis. To produce the diagram

\footnotetext{
${ }^{7}$ In a Fish War off Newfoundland, a deal between Canada and Spain was struck in which Canadian fishermen received quota which were lower than in an earlier agreement broken by the Spanish. A real world example of the patient being exploited by the impatient? Another example can be found in Kennedy [1987, p.7] where the Australian government considered lowering Australian quota in case Japanese catch was not curtailed sufficiently, this being deemed in the interest of Australia itself.

${ }^{8}$ I thank Christian Cordes for reminding me of this fact.

${ }^{9}$ Note that Rubinstein [2003] observes that the criticism leading to a rejection of standard constant discount utility functions can easily reject hyperbolic discounting as well.
} 
below we computed for different values of $D \geq 0$ the same three numbers namely $\varphi_{D}-\omega_{D}$ (in blue), $\varphi_{D}-\psi_{D}$ (in black) and $\chi_{D}-\omega_{D}$ (in red). ${ }^{10}$ Recall that the more patient agent has a lower parameter $D$.

The results for hyperbolic discounting are quite comparable to the results for exponential discounting. Let us call the intersection point of the bluegreen curve with the horizontal axis $D_{\text {right }}$, the corresponding point for the black curve $D_{\text {middle }}$, and the intersection point of the red curve $D_{\text {left }}$.

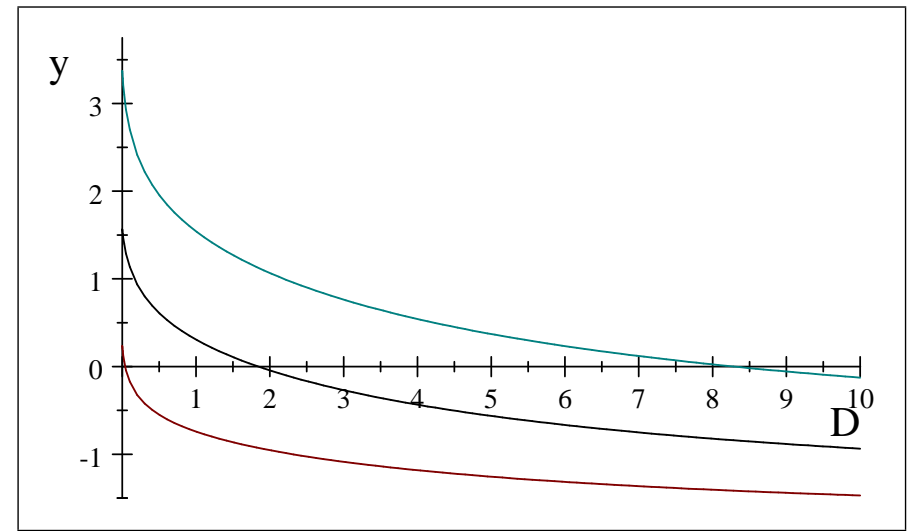

We can now give the following overview with respect to $D \geq 0$.

- Very impatient $\left(D>D_{\text {right }}\right)$ : The corresponding matrix is $M_{V V}$. Each player has a dominant action, namely to defect; the socially defective choice induces an equilibrium which Pareto-dominates the socially cooperative one. The fish stock will be depleted.

- Impatient $\left(D_{\text {middle }}<D<D_{\text {right }}\right)$ : The corresponding matrix is $M_{I I}$. There is little hope of reconciling the agents' interests with sustainability of the fish stock in this PD. The fish stock will be depleted (in all likelihood).

- Moderately patient $\left(D_{\text {left }}<D<D_{\text {middle }}\right)$ : The corresponding matrix is $M_{M M}$. In this $\mathrm{CH}$, it is optimal that one agent always catches with restraint, while the other one catches without. The long run fish stock can be computed easily as $\mu=\frac{1}{2}$.

- Patient $\left(D<D_{\text {left }}\right)$ : The corresponding matrix is $M_{P P}$. In the resulting PG, the agents care for high long-term yields and therefore for high long-term fish stocks. Hence, there is no contradiction between self-interest and sustainability.

\footnotetext{
${ }^{10}$ To speed up computations, we used several finite evaluation periods $T$ (instead of $\infty$ ). For $T=50,75,100,150$ qualitatively similar diagrams were obtained.
} 
As the reader may confirm, the same types of social dilemmas appear for the same ranges of patience. Again, the matrices relevant for the asymmetric time-preferences case are $M_{P M}, M_{P I}, M_{P V}, M_{M I}, M_{M V}$ and $M_{I V}$. For space considerations, we refer to the previous section for details regarding those 6 matrices.

Remark 1 Details regarding the type of discounting do matter in practice, for instance, where precisely intersection points of the three curves are (if there exists one). Both methods of evaluating the future may give conflicting answers as to which games are possible, e.g., we have constructed examples in which a curve intersects the horizontal axis in exponential discounting, while its hyperbolic counterpart does not. However, both types of discounting allow the same type of conceptual analysis.

Remark $2 A$ whole range of games may arise in one and the same common pool resource game due to different degrees of patience on the part of the agents. This range may very well contain the $P D$ as a special case, but also e.g., the CH, PG or the Bully Game. Moreover, we found that not every game possible is a social dilemma.

Remark 3 Monotonicity with respect to $\beta(D)$ of any curve $\varphi_{\beta}-\omega_{\beta}, \chi_{\beta}-\omega_{\beta}$ or $\varphi_{\beta}-\psi_{\beta}\left(\varphi_{D}-\omega_{D}, \chi_{D}-\omega_{D}\right.$ or $\left.\varphi_{D}-\psi_{D}\right)$ implies that such a curve intersects the $\beta$-axis at most once.

\section{The resource and behavior}

We generated an example which seems not too far off from some other work both from empirics and theory with respect to which predictions can be made under which levels of patience. In a theoretical model analyzing optimal management of North Sea herring, Maroto \& Moran [2008] find a similar relationship between patience and sustainability. Discount factors below 0.71 , i.e., great impatience in our terminology, lead to 'rational' overfishing and extinction; discount factors between 0.72 and 0.85 , i.e., impatience, induce periodical flirting with extinction and return to high fish stock levels is unlikely; for discount factors between 0.86 and 0.94, i.e., moderate impatience, extinction is less likely, yet full recovery is unlikely as well; only lower discount factors induce sustainability.

Bjørndal [1988] shows for the North Sea herring fishery that a discount rate above 0.53 ( $\beta \approx 0.65$, i.e., a high degree of impatience) implies the 'tragedy' as an 'optimal' outcome, whereas a rate below $0.12(\beta \approx 0.89$, i.e., a high degree of patience) induces high fish stocks and corresponding high sustainable landings.

Hillis \& Wheelan [1994] report discount rates of fishermen (and political institutions) between $0.25(\beta \approx 0.80)$ and $0.40(\beta \approx 0.71)$. In the numerical 
example of our model, this qualifies as impatient, with one extreme value very close to very impatient and the other one rather close to moderately impatient. This would place the discounted games in the range of a Prisoners' Dilemma $\left(M_{I I}\right)$ with the extremes close to $M_{V V}$ and $M_{M M}$ (a CH).

\subsection{Reactivity of the resource system}

We see eight parameters in the case analyzed in the previous section, namely,

$$
\begin{gathered}
a, b, c, d \\
n_{1}, n_{2}, q, \underline{m}
\end{gathered}
$$

$P S$ denotes the parameters pertaining to the payoff structure, and $R S$ to the resource system. Having eight parameters governing several equations linking the various relationships, one might get lost which parameter causes which effect. We will come back to the complexity of the model in Section 5 . However, we made one striking observation with respect to the parameters in $R S$ and we devote the remainder of this section to it.

To generate the diagram for the discounting case below, we only increased the minimal fish stock, i.e., $\underline{m}=0.1$. The diagram should be compared to the diagram in Subsection 3.1.

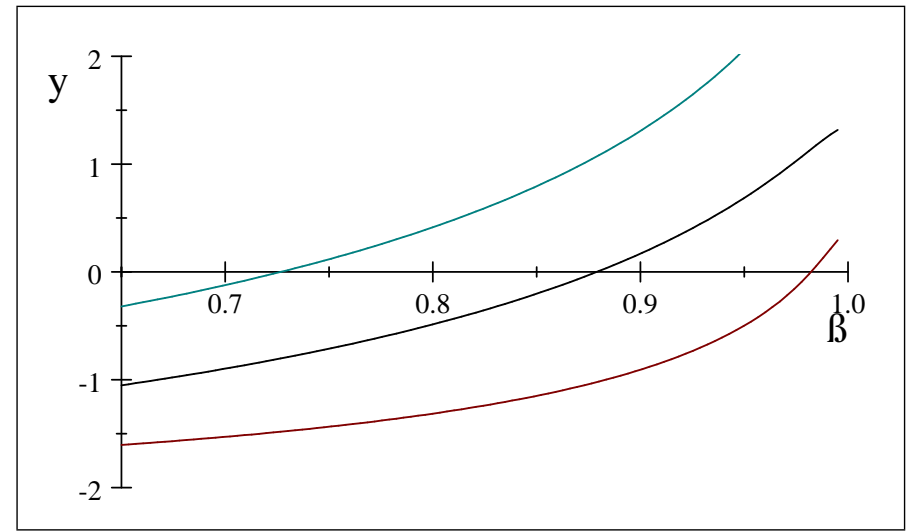

Qualitatively, this diagram is similar to the one corresponding to the case for the lower-level minimal fish stock, $\underline{m}=0$, but there is a noticeable difference. First, $\beta_{\text {left }}$ moves to the right. So, the very worst conditions on the game change to the second worst, i.e., the $\mathrm{PD}$, only for a higher value $\left(\beta_{\text {left }}\right)$ of the discounting parameter. This effect is exemplary for the other changes. The PD transforms into the $\mathrm{CH}$ at a higher value $\left(\beta_{\text {middle }}\right)$, and the latter one into the $\mathrm{PG}$ at a higher value $\left(\beta_{\text {right }}\right)$.

There are two other parameter constellations influencing the speed in which the system reacts to overfishing at high resource stock levels. One parameter is $q$, where a high value means that the (early) effects of unrestrained fishing on the fish stock are more 'damped'. The other one depends 
on the interplay between $n_{1}$ and $n_{2}$. If both decrease while keeping their difference fixed, the system becomes more reactive. We have examined the effects of these constellations on the functions and graphs used for analysis in the preceding section, and we found that a higher (lower) $q$ and higher (lower) $n_{1}$ and $n_{2}$ while keeping $n_{1}-n_{2}=1$ cause the three curves in the graph to shift to the right (left).

For hyperbolic discounting we checked the same three effects in isolation, i.e., changes in minimal fish stock $\underline{m}$, changes in the damping parameter $q$ and changes in $n_{1}$ and $n_{2}$. In all three cases mentioned the curves changed in the direction expected, i.e., in the opposite direction of the movements of the corresponding curves in the exponential discounting case. For the sake of brevity we only present the three curves belonging to the case where the minimal fish stock is increased to $\underline{m}=0.1$. Note that all intersection points move to the left.

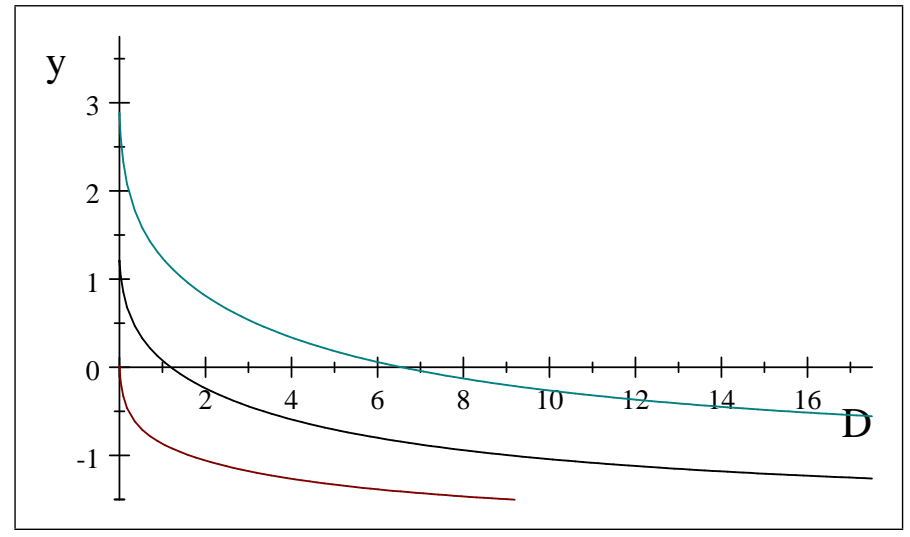

How are these observations to be interpreted? The reason for the shifts mentioned seems that if the consequences of catching without restraint, i.e., by the use of the technology harmful to the resource, are felt earlier by the agents because the resource system reacts more quickly, or more strongly, then the thresholds relevant for our analysis concerning the discounting parameters occur at lower levels. This gives the following insight.

Remark 4 The more (less) reactive the fish stock due to overfishing in the high fish-stock-levels range, the lower (higher) the discount parameters for which the agents 'come to their senses'.

Chances for sustainability worsen for shifts to the right if one assumes the discount parameter $\beta$ to be drawn from some fixed distribution. Let for instance $\beta$ be uniformly distributed on the interval found by Hillis \& Wheelan [1994], i.e., the interval $[0.71,0.8]$. Assuming perfectly symmetric timepreferences, in the original setting game most likely to be played is a Prisoners' Dilemma, but the left boundary of the interval is close to $M_{V V}$ and the right one is not very far from $M_{M M}$, which corresponds to a Chicken 
Game. A shift to the right of all curves implies there now exists a positive probability that the game played is $M_{V V}$, hence the new situation is to be regarded as worse than before the shift. Contrary, a sufficiently large shift to the left would induce a positive probability of a $\mathrm{CH}$ being played, improving the chances for sustainability considerably.

\subsection{The state of the resource}

Remark 4 suggests a potential for framing in the sense used in behavioral economics (Kahneman \& Tversky [1984], \& Tversky Kahneman \& Tversky [1981,1986], Schelling [1984]), i.e., to communicate the most pessimistic scenarios. The graver the problem is perceived, the more likely the agents behave in the interest of sustainability, cf., e.g., Joosten [2007b]. The following caveat is to be kept in mind. Hillis \& Wheelan [1994] attribute the (in their view) impatience of fishermen to the great uncertainty the latter perceive about future landings (see also Döring [2006]). ${ }^{11}$ Several factors may contribute to (perceived) uncertainty in a real-world common pool resource system, for instance stochastic resource dynamics, weather or climatic conditions, spatial aspects, Allee effects, the number of agents, legal and institutional settings of the resource system in isolation or even in combination. In a system close to exhaustion the future might be more heavily discounted (receive a much lower weight), than in a system in which the resource is available at maximum capacity. ${ }^{12}$ This would complicate any mission towards recovery to high fish stocks considerably, or alternatively, aggravate the danger of exhaustion enormously.

In the contributions mentioned, Irish See fishermen are called 'impatient'. A 'benchmark' for patience clearly is lacking. If discount rates of $25 \%$ to $40 \%$ of Irish See fishermen are compared to the long-term 'riskfree' interest rates on the capital market (well below 10\%), then indeed they may be called impatient. However, recent work suggests that their time-preferences ${ }^{13}$ might not be too far off from those of people in other

\footnotetext{
${ }^{11}$ See Deutsch [1978] for one psychological interpretation, namely the scepticism that 'good' own behavior will lead to the desired outcome. Dawes [1980] and Edney [1980, 1981] offer another, namely the expectation of nonreciprocation of cooperative behavior (cf., e.g., Brann \& Foddy [1987]). Trust might be an issue too, cf., e.g., Messick et al. [1983], Brann \& Foddy [1987]. Döring \& Egelkraut [2008] suggest that fishermen's long-term uncertainty should be reduced to avert the threat of aggressive short-term behavior. Bjørndal \& Gordon [1993] observe investment behavior in the Norwegian fishing fleet even under low rates of return suggesting an absence or reduction risk due to the nature of the fishery at hand (cf., e.g., Boncoeur et al. [2000] and Edney \& Harper [1978]). Hannesson [1997] defends a discount rate between $5-10 \%$ in case the number of competitors is particularly low which induces cooperation (see also Komorita \& Parks [1996] on analogous findings).

${ }^{12}$ In our model, this might imply that we would have variable $\beta$ 's (or $D$ 's) depending on the level of the present fish stock, i.e., $\beta_{t}=f\left(\mu_{t}\right)$ (or $D_{t}=g\left(\mu_{t}\right)$ ) with $f$ nonincreasing ( $g$ nondecreasing) in the argument.

${ }^{13} \mathrm{We}$ are aware of the criticism spelled out in the overview of Frederick et al. [2002].
} 
professions or positions (cf., e.g., Warner \& Pleeter [2001] ${ }^{14}$ or Harrison et al. $\left.[2002]^{15}\right)$.

\section{Generalizability}

To demonstrate the generalizability of the approach, we analyze a dilemma characterized by an interaction matrix given by (3) with $c<a<b<d$, i.e., a Stag Hunt or Assurance Game (cf., e.g., Skyrms [2004], Sen [1967]]). This structure might arise here if the environmentally harmful technology has strong positive externalities with itself and mild externalities with the other, while being very costly in operating. So, if both players happen to choose that technology, the benefits outweigh the costs by far in every stage game. However, if only one player chooses this technology and the other one does not, then high costs are borne by the former but insufficient benefits come in to compensate. So, this technology can be viewed as very risky, both the highest and the lowest one-shot payoffs are associated to it.

However, we are not merely interested in the stage games associated with the resource game, we are interested in the discounted rewards of the game which are subject to two different simultaneous effects. Firstly, the fish stock deteriorates as time progresses if the environmentally damaging technology is adopted; the more adopters the quicker the stock goes down. Secondly, the manner in which the agents discount has influences the same type of matrix differently for each entry.

For the discussion to follow, let us define the following family of matrices and describe the connections between them. The reason is that as in the analysis presented in Section 3, we construct a sequence of relevant matrices following from increasing patience on the part of the agents.

$$
\begin{gathered}
M_{1}=\left[\begin{array}{cc}
2,2^{*} & 3,1 \\
1,3 & 4,4^{*}
\end{array}\right], M_{2}=\left[\begin{array}{cc}
3,3^{*} & 2,1 \\
1,2 & 4,4^{*}
\end{array}\right], M_{3}=\left[\begin{array}{cc}
4,4^{*} & 2,1 \\
1,2 & 3,3^{*}
\end{array}\right], \\
M_{4}=\left[\begin{array}{cc}
4,4^{*} & 3,1 \\
1,3 & 2,2
\end{array}\right], M_{5}=\left[\begin{array}{cc}
4,4^{*} & 3,2 \\
2,3 & 1,1
\end{array}\right], M_{6}=\left[\begin{array}{cc}
2,2^{*} & 4,1 \\
1,4 & 3,3
\end{array}\right], \\
M_{7}=\left[\begin{array}{cc}
3,3^{*} & 4,1 \\
1,4 & 2,2
\end{array}\right], M_{8}=\left[\begin{array}{cc}
3,3^{*} & 4,2 \\
2,4 & 1,1
\end{array}\right] .
\end{gathered}
$$

In the matrices above, a star denotes a pair of rewards associated with an equilibrium. So, the first three games have two equilibria, full-restraint or

\footnotetext{
So, it is not wise to attribute too much confidence even to these results.

${ }^{14}$ They report estimated discount rates of 0.104 for officers in the US army around 1992, and 0.354 for enlisted personnel (later corrected to 0.173 ), well below the lower bound in Hillis \& Wheelan [1994], yet above the officers' rates.

${ }^{15}$ Reporting a $90 \%$ confidence interval of $[0.2726,0.2903]$ for discount rates of the general public in Denmark.House owners (0.2561) and non-owners (0.3167), less educated (0.3098) and more educated (0.2059), skilled (0.2573) and unskilled (0.3143) differ.
} 
full-exploitation; the other ones only one, full-restraint. $M_{1}$ and $M_{2}$ are Stag Hunts, $M_{3}$ is not. In the remaining five games the choice of the sustainable technology dominates the alternative. Hence, in those games game theory predicts full-restraint and sustainable fish stocks at maximum level. Note however, that $M_{6}$ is a Prisoners' Dilemma.

Observe that $M_{2}$ can be obtained by switching the spots of ordinal utilities 2 and 3 in $M_{1}$. We will call such a transition from one to another matrix involving at most two consecutive relative utilities (for each player) as nondegenerate. Note that $M_{3}$ can be obtained from $M_{1}$ by two non-degenerate transitions (via $M_{2}$ ), but that the direct transition is degenerate. A transition is symmetric if the same changes occur for both players. Again the transition from $M_{1}$ to $M_{2}$ is a symmetric one, because for both players the 2 and 3 exchange their places.

A sequence of transitions is admissible in the present framework if they satisfy the following properties.

- The relative ordering of the off-diagonal entries do not change.

- Each top-left entry can never decrease and each bottom-right entry can never increase.

To illustrate the first point of admissibility, observe the top-right entry in $M_{1}$ has entry $(3,1)$ and the left-bottom entry is $(1,3)$. So, for Player 1 the top-right reward is better than the bottom-left reward. Matrix $M_{2}$ can be obtained from $M_{1}$ by an admissible transition. Observe now that again for Player 1 the top-right reward, i.e., 2, is better than the bottom-left reward, i.e., 1. The bottom-right entries did not change and both top-left entries increased. Hence, the transition is admissible.

The following overview represents the 'universe' created by all possible non-degenerate, symmetric, admissible matrices emanating from $M_{1}$

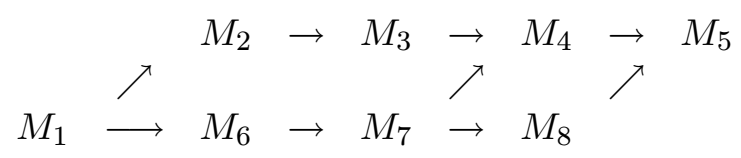

\subsection{The resource games for increasing patience}

The crucial point now is to establish where a sequence begins and where it ends in this little 'universe'. There may be little surprise that any sequence starts in $M_{1}$ but it is not trivial where such a sequence ends. For instance, if $\underline{m}=1$ or alternatively if this lower bound for the fish stock is sufficiently high, the sequence may start and terminate in the same matrix. We determine the terminating matrix by looking at ${ }^{16}$

$$
M_{e n d}=\left[\begin{array}{cc}
\varphi_{1}, \varphi_{1} & \chi_{1}, \psi_{1} \\
\psi_{1}, \chi_{1} & \omega_{1}, \omega_{1}
\end{array}\right]
$$

\footnotetext{
${ }^{16}$ For the sake of brevity, we only refer to the exponential discounting approach.
} 
As both players have symmetric time-preferences, one of the matrices above is a candidate as an end matrix for the sequence of transitions, but only one qualifies depending on the entire set of parameters of the resource system.

Now, suppose $M_{e n d}=M_{8}$, then a unique sequence exists from $M_{1}$ involving three transitions, comparable in spirit to the sequence appearing in Section 3. There are three critical $\beta$ 's changing the structure of the game. However, if $M_{\text {end }}=M_{2}$, there exists only one transition for one $\beta$. On the other hand, if $M_{\text {end }}=M_{4}$ or $M_{\text {end }}=M_{5}$ multiple sequences of different lengths exists. Here, the number of matrices in the sequence can be taken as a measure of length. In the above, there exist two sequences of length 1 , two of length 2 , three of length 3 , and three sequences of length 4 .

Observe furthermore that of the sequences leading to $M_{5}$ one can be regarded as more dangerous to the resource than the two others. There exists one sequence $M_{1} \rightarrow M_{2} \rightarrow M_{3} \rightarrow M_{4} \rightarrow M_{5}$, and two alternatives containing $M_{6}$. The former sequence requires two utility transitions caused by increased patience on the part of the agents for the resource to be safe from exhaustion, because only at $M_{4}$ the game has the environmentally safe outcome as its unique equilibrium. In the latter two sequences, already after one transition caused by increased patience of the agents the resource may be regarded as safe from exhaustion assuming rationality of agents and the impossibility of making binding agreements.

\subsection{Reducing complexity}

Complexity of the case above is not too cumbersome and therefore it serves to demonstrate the approach in full. However if we drop the requirement of symmetry regarding the transitions matters become much more involved. Even if we assume the starting and ending matrices to coincide for the case of symmetric and asymmetric transitions, the number of sequences and the number of matrices to be examined increase very quickly. Suppose in the symmetric case we need just one transition from starting to ending matrix, in the asymmetric case we already need two. Similarly, two symmetric transitions require an analysis of six potential asymmetric ones; three induce 20 , four induce 70 . In that case the small universe might ${ }^{17}$ explode to a universe with 286 games more.

The first relief to the reader may come from the observation that for the entire universe of possible transitions considerable efforts may be required indeed, but for one specific setting matters are comparable to the efforts of establishing an overview of the universe of transitions in Section 3.

Furthermore, we need not make a distinction between all eight matrices in the analysis above, or the rapidly increasing number of matrices for transitions. What is crucial is whether rational agents will use the envi-

\footnotetext{
${ }^{17}$ We did not check whether the 286 additions are unique, hence the might.
} 
ronmentally harmful technology in equilibrium. Recall that the structure relevant for a game-theoretical analysis on the signs of $\varphi_{\beta_{1}}-\psi_{\beta_{1}}, \chi_{\beta_{1}}-\omega_{\beta_{1}}$, $\varphi_{\beta_{2}}-\psi_{\beta_{2}}$, and $\chi_{\beta_{2}}-\omega_{\beta_{2}}$. Hence, the total universe of game-theoretically and sustainability-related relevant transitions consists of at most four.

For instance, $M_{1}, M_{2}$ and $M_{3}$ have two pure Nash equilibria, hence sustainability is endangered as one equilibrium involves the mutual choice of the harmful technology. Furthermore, a third Nash equilibrium in mixed strategies exists which we did not address in the above as we focus on pure equilibria and on ordinal utilities. A mixed equilibrium still endangers the resource as with positive probability both agents adopt the harmful technology. For the other matrices in the overview, the use of the harmful technology is dominated by the use of the harmless one. Hence, by structuring the analysis around the game-theoretically important transitions, an enormous reduction in complexity is achieved regarding the total overview, and for specific cases analysis is less involved anyway.

\section{Conclusions}

In earlier work on Small Fish Wars (Joosten [2007a,b,c]) we used the average reward criterion for a number of reasons. One being that the average reward criterion leads to a consistent mapping for given behavior of what the common-pool resource yields in the long run and how the agents evaluate their revenues. Furthermore, it is evident that under discounting, any immediate advantage seems to be preferred to any environmental or economic catastrophe sufficiently far away in the future, cf., e.g., Heal [1998], Weitzman [1998, 2001]. Schelling [1995] stresses that discounting is appropriate within a generation, not between generations, see also e.g., Rabl [1996]. The standard interpretation of using the average reward criterion is that agents are very patient, i.e., future payoffs are equivalent to current ones.

Here, we study the situation in which patience is 'finite', agents value a payoff now differently from the same payoff in the future, i.e., they prefer a payoff now to the same in the future. We therefore analyze a Small Fish War using exponential discounting and hyperbolic discounting, two of the most common methods of comparing intertemporal payoffs. Technology choice is assumed a once-and-for-all decision, or alternatively for a sufficiently long period of time. This may occur for instance, if technologies are expensive and difficult to get rid of, or involve large sunk or switching costs. Both types of discounting allow a similar qualitative analysis.

In an example examined for expository purposes we find for both that the interaction of agents in a resource system induces several different 'subjective' games depending on the level of patience of the agents. For a group of impatient, profit maximizing agents it might be perfectly rational to deplete the fish stock completely and as quickly as possible. 
However, a 'tragedy of the commons' is not unavoidable as several other scenarios may occur. For different ranges of patience and assuming sufficiently symmetric time-preferences, the games to occur are for instance, a Prisoners' Dilemma (PD), a Chicken Game $(\mathrm{CH})$ or a Privileged Game $(\mathrm{PG})$. Very impatient agents play a game endangering the resource, as equilibrium behavior implies acceptance of the environmentally harmful technology. More patient agents play a PD and the problem here is only slightly less grave, but 'rational' technology choice still favors the harmful one.

Considerably better prospects for the resource arise in the two other games. Even more patient agents play a $\mathrm{CH}$, a game with two equilibria. In each equilibrium one agent uses the harmless technology, and the other the harmful one. Both pure equilibria induce long run fish stocks below maximum, but well above minimum stock level. The real problem here is coordination, who will take which technology? The final social dilemma is a $\mathrm{PG}$, which is good for the resource as equilibrium behavior implies mutual choice of the environmentally sound technology.

Sufficiently asymmetric time-preferences yield an entirely different picture. In five out of six games arising from the same example, the more patient agent chooses the harmless technology, whereas the opponent chooses the harmful one. The less patient agent obtains the most favored outcome in all of these five cases, the patient one obtains the next to worst outcome in all six cases. Again the effects on the resource are moderate, full exhaustion is curbed, yet the resource is not at its maximum stock either. In the remaining game, both exploit the resource ruthlessly.

To demonstrate that the method of analysis can be applied universally, we analyze another social dilemma, a Stag Hunt, in full. Instead of focussing on one particular setting, we examine all possible games which may arise from this particular Stag Hunt in a Small Fish War under discounting. Starting fairly cautiously by allowing only non-degenerate, symmetric and admissible transitions, we find a 'universe' of eight of such games.

The dynamics of the resource determine a unique sequence of games connecting the Stag Hunt with a so-called end game. Alternative sequences may exist connecting the Stag Hunt and this end game, but only one is relevant. The interpretation then is that the sequence represents the collection of games arising by letting the agents become more and more patient as in the example studied for expository purposes.

The general case proved to be very complex, as dropping symmetry for instance, caused our eight-game universe to explode to more than 280 games. We recommend therefore to analyze only the transitions relevant for game theoretical predictions which also constitute the relevant cases for sustainability of the resource. Furthermore, we propose, if possible, to only tackle well-described settings such that indeed a unique sequence of games within this possibly very large universe is to be expected.

Elinor Ostrom and co-workers lament the 'indiscriminate use of three 
metaphors ... to predict suboptimal use and/or destruction of the resource' (see Ostrom et al. [1994], and also Ostrom [1990]). The three influential metaphorical models meant are the tragedy of the commons, the Prisoners' Dilemma $^{18}$ and the logic of collective action ${ }^{19}$. Equilibrium behavior induces a situation to be regarded as inferior to a possibly non-equilibrium alternative in the sense that had somebody dictated the latter, this would be preferred by all. This describes a social dilemma.

Two frequently parroted theoretical solutions to common pool resource problems are quite simplistic and even contradictory. One recommends a Hobbesian 'Leviathan', i.e., a government with major coercive powers (cf., e.g., Ophuls [1973, p. 228], to induce the alternative preferred by all as the 'only way' (cf., Ostrom [1990]). The other proposes private property rights, e.g., Demsetz [1967], Johnson [1972] as the 'only way', e.g., Smith [1981].

Remarkably, game theory has a hard time explaining the many nonfailing dilemmas collected by the Ostrom and cooperators. Much of this group's work (e.g., Ostrom et al. [1994], Ostrom [1990,2005]) revolves around the theme that a rich array of institutional settings can curb the threat of the tragedy. Among the aspect that may be helpful in generating favorable (pre)conditions for sustainable high extraction rates are communication, repeated interaction, monitoring, possible punitive actions, voluntary rules, trust, various institutional designs.

To put our contribution into a perspective regarding the above. We have shown that quite a few games may arise in the context of our model of a specific common pool resource game. Some are Prisoners' Dilemmas, many are not. Some have the characteristics of a tragedy of the commons, others lead to a sustainable resource under equilibrium behavior.

Information is crucial in assessing the sustainability of a certain resource under equilibrium behavior. Suppose we know everything about the resource and the agents, then we know the exact game to be played. However, if we have complete information regarding the resource, yet incomplete information regarding the time-preferences of the agents involved, we must do with a unique sequence of games, of which just one will be played. If we know the current state of the resource and we possess information on what ruthless exploitation can do to it in the end, yet can not entirely describe what happens in between, we must establish a possibly very large universe of games, of which a unique one will be played, not known in advance.

This poses problems of increasing magnitude to a field researcher as well

\footnotetext{
${ }^{18}$ Dawes $[1973,1975]$ is reputed to be the first scholar to show similarities between the prisoner's dilemma and the tragedy of the commons. Similarity does not imply equivalence as many, e.g., Godwin \& Shepard [1979], Kimber [1981], tried to demonstrate.

${ }^{19}$ Olson $[1965$, p.2] challenged communis opinio at that point in time that group benefit would inevitably trigger collective action to achieve that benefit: "unless there is coercion or some other special devise to make individuals act in their common interest, rational self-interested individuals will not act to achieve their common or group interests".
} 
as to a benevolent ${ }^{20}$ authority examining whether or not to intervene in the game or not by designing rules to be adhered to or alternatively by assigning property rights. Which game will (most likely) be played? For a scientist to judge whether or not observed behavior corresponds to equilibrium behavior or not, the game must be known. For this purpose, not only relevant knowledge about the resource dynamics under (over)exploitation should be available, but also about how the agents evaluate future payoffs.

Perhaps the information on the part of the agents is better than the information the authorities possess. So one might want to advise authorities to keep out. However, even the agents themselves might not entirely know the game to be played. For instance, if they know how they evaluate the strategic interaction themselves, they might be lacking the knowledge about the time preferences of their opponents. This might endanger the unenforced installment of institutional settings to avoid the tragedy of the commons.

We also found equilibrium behavior which is not necessarily bad for sustainability, the resource survives at considerable higher stock levels than under full exploitation, yet equilibrium yields quite unegalitarian outcomes. With the latter we mean that one agent receives a much more preferred outcome than his opponent. We found this outcome in a Chicken Game setting for sufficiently similar time-preferences, but rather frequently in games arising under sufficiently dissimilar time-preferences. In almost all asymmetric preference games, the more patient agent will be exploited by the more impatient one. In the Chicken Game coordination is an issue, in the former as well as in the latter games inequality is, both for the agents as it complicates reaching agreements, or intervention by benevolent authorities.

Further research must show what the effects of 'perverse' price-scarcity dynamics for the present model(s) are. In a series of papers, Courchamp and co-workers (c.f., e.g., Berec et al. [2006], Courchamp et al. [2006], Hall et al. [2008]), have highlighted rarity value, a slightly counterintuitive mechanism under which unit prices increase without a bound for increased scarcity of the resource. Such a setting will in general aggravate the usual problems of a common pool resource system. Once a species becomes scarce, its increased value make the propensity to exploit it grow, which increases its scarcity etc.

In earlier work (Joosten $[2007 \mathrm{~b}, \mathrm{c}]$ ), we hypothesized that we might observe such an effect actually occurring: the blue fin tuna. Prices of blue fin tuna have broken a considerable number of records in the past few years, yet demand is not declining. We also showed that infinite patience in the presence of rarity value need not help at all in sustaining the resource. We know that myopic time preferences do not help in general and that infinite patience is not helpful either. Perhaps intermediate ranges of patience may

\footnotetext{
${ }^{20}$ Benevolent as in wishing to do 'justice' to the agents as well as desiring to preserve the resource with as little intervention as possible.
} 
lead to sustainable outcomes, but a full examination is still pending.

\section{$7 \quad$ References}

Ainslie G, 1974, Impulse control in pigeons, J Experimental Animal Behav 21, 485-489.

Ainslie G, 1975, Specious rewards: a behavioral theory of impulsiveness and reward, Psychological Bulletin 82, 463-496.

Ainslie G \& R Herrnstein, 1981, Preference reversal and delayed reinforcement, Animal Learning \& Behav 9, 476-482.

Bennet P, 1998, Confrontation analysis as a diagnostic tool, Eur J Oper Res 109, 465-482.

Berec, L, E Angulo \& F Courchamp, 2006, Multiple Allee effects and population management, Trends Ecol \& Evol 22, 185-191.

Bjørndal T, 1988, The optimal management of North Sea herring, J Environ Econ 85 Management 15, 9-29.

Bjørndal T \& DV Gordon, 1993, The opportunity cost of capital and optimal vessel size in the Norwegian fishing fleet, Land Econ 69, 98-107.

Boncoeur J, L Coglan, B Le Gallic \& S Pascoe, 2000, On the (ir)relevance of rates of return measures of economic performance to small boats, Fisheries Res 49, 105-115.

Brann P \& M Foddy, 1987, Trust and the consumption of a deteriorating common resource, J Conflict Resolution 31, 615-630.

Campbell R, 1985, Background for the uninitiated, in: "Paradoxes of Rationality and Cooperation" (R Campbell \& L Sowden, eds.), UBC Press, Vancouver, pp. 3-41.

Courchamp F, E Angulo, P Rivalan, RJ Hall, L Signoret \& Y Meinard, 2006, Rarity value and species extinction: The anthropogenic Allee effect, PLOS Biol 4, 2405-2410.

Cross JG \& MJ Guyer, 1980, "Social Traps", University of Michigan Press, Ann Arbor, Michigan.

Dawes RM, 1973, The Commons Dilemma game: An N-person mixedmotive game with a dominating strategy for defection, ORI Research Bulletin 13, 1-12.

Dawes RM, 1975, Formal modiels of dilemmas in social decision making, in: "Human Judgment and Decision Processes: Formal and Mathematical Approaches" (MF Kaplan \& S Schwartz, eds.), Academic Press, NY, pp. 87-108.

Dawes RM, 1980, Social Dilemmas, Annual Rev Psychology 31, 169-193. Demsetz H, 1967, Toward a theory of property rights, Am Econ Rev 62, 347-359

Deutsch MD, 1978, "The Resolution of Conflict", Yale University Press, New Haven, Connecticut. 
Döring R, 2006, Investing in natural capital-the case of fisheries, in: " 2005 North American Association of Fisheries Economists Forum Proceedings" (Sumaila, UR \& AD Marsden, eds.), Fisheries Centre, Univ BC, Vancouver, Canada, pp. 49-64.

Döring R \& TM Egelkraut, 2008, Investing in natural capital as management strategy in fisheries: The case of the Baltic Sea cod fishery, Ecol Econ 64, 634-642.

Dudley L, 2000, The rationality of revolution, Econ Governance 1, 77-103. Dudley L \& U Witt, 2004, Arms and the Man: World War I and the rise of the welfare state, Kyklos 57, 475-504.

Edney JJ, 1980, The commons problem: alternative perspectives, Amer Psychologist 35, 131-150.

Edney JJ, 1981, Paradoxes in commons: scarcity and the problem of equality, J Community Psychology 9, 3-34.

Edney JJ \& CS Harper, 1978, The effects of information in a common resource management problem: a social trap analysis, Human Ecology 6, 387-395.

Frederick S, G Loewenstein \& T O'Donoghue, 2002, Time discounting and time preference: a critical review, J Econ Lit 40, 351-401.

Fudenberg D \& E Maskin, 1986, The Folk Theorem in repeated games with discounting or with incomplete information, Econometrica 54, 533-554. Godwin RK \& WB Shepard, 1979, Forcing squares, triangles and ellipses into a circular paradigm: The use of the Commons Dilemma in examining the allocation of common resources, Western Political Quarterly 32, 265277.

Gordon HS, 1954, The economic theory of a Common-Property Resource: The Fishery, J Pol Econ 62, 124-142.

Guyer M \& Rapoport A, 1966, A taxonomy of 2 x 2 games, General Systems 11, 203-214.

Hall RJ, EJ Milner-Gulland \& F Courchamp, 2008, Endangering the endangered: The effects of perceived rarity on species exploitation, Conservation Letters 1, 75-81.

Hamburger H, 1973, N-person prisoner's dilemma, J Math Psychology 3, $27-48$.

Hannesson R, 1997, Fishing as a supergame, J Environmental Econ $\&$ Management 32, 309-322.

Hardin G, 1968, The tragedy of the commons, Science 162, 1243-1248.

Harrison GW, MI Lau \& MB Williams, 2002, Estimating individual discount rates in Denmark: A field experiment, Amer Econ Rev 92, 16061617.

Heal GM, 1998, "Valuing the Future: Economic Theory and Sustainability", Columbia University Press, New York.

Heckathorn DD, 1996, The dynamics and dilemmas of collective action, Amer Sociolog Rev 61, 250-277. 
Hillis JF \& BJ Wheelan, 1994, Fisherman's time discounting rates and other factors to be taken into account in planning rehabilitation of depleted fisheries, in: "Proceedings of the 6th Conference of the International Institute of Fisheries Economics and Trade" (M Antona, J Catanzano, JG Sutinen, eds.), IIFET-Secretariat Paris, pp. 657-670.

Hobbes T, 1651, "Leviathan (or The Matter, Forme and Power of a Common Wealth Ecclesiasticall and Civill)", Clarendon, Oxford.

Hume D, 1739, "A Treatise of Human Nature", Clarendon, Oxford.

Johnson OEG, 1972, Economic analysis, the legal framework and land tenure systems, J Law $\&$ Econ 15, 259-276.

Joosten R, 2005, A note on repeated games with vanishing actions, Intern Game Theory Rev 7, 107-115

Joosten R, 2007a, Small Fish Wars: A new class of dynamic fisherymanagement games, ICFAI J Managerial Econ 5, 17-30.

Joosten R, 2007b, Small Fish Wars and an authority, in: "The Rules of the Game: Institutions, Law, and Economics" (A Prinz, AE Steenge \& J Schmidt, eds.), pp. 131-162.

Joosten R, 2007c, Patience, Fish Wars, rarity value \& Allee effects, Papers Econ $\&$ Evol \# 0724, Max Planck Institute of Economics, Jena.

Joosten R, T Brenner \& U Witt, 2003, Games with frequency-dependent stage payoffs, Int J Game Theory 31, 609-620.

Kahn H, 1965, "On Escalation: Metaphors and Scenarios", Praeger, NY.

Kahneman D \& A Tversky, 1984, Choices values and frames, Amer Psychologist 39, 341-350.

Kelly HH \& JW Thibaut, 1978, "Interpersonal Relations", Wiley, NY.

Kennedy JOS, 1987, A computable game theoretic approach to modelling competitive fishing, Marine Resource Econ 4, 1-14.

Kimber R, 1981, Collective Action and the fallacy of the Liberal Fallacy, World Politics 33, 178-196.

Komorita SS \& CD Parks, 1994, "Social Dilemmas", Brown \& Benchmark's Social Psychology Series, Brown \& Benchmark, Madison WI.

Koopmans TC, 1960, Stationary ordinal utility and impatience, Econometrica 28, 287-309.

Liebrand WBG, 1983, A classification of social dilemma games, Simulation \& Games 14, 123-138.

Loewenstein G \& D Prelec, 1992, Anomalies in intertemporal choice: evidence and an interpretation Quart J Econ 107, 573-598.

Loewenstein G \& R Thaler, 1998, Anomalies: intertemporal choice, $J$ Econ Perspectives 3, 181-193.

Levhari D \& LJ Mirman, 1980, The great fish war: An example using a dynamic Cournot-Nash solution, Bell J Econ 11, 322-334.

Marwell G \& P Oliver, 1993, "The Critical Mass in Collective Action: A Micro-Social Theory", Cambridge University Press, Cambridge, UK. 
Maroto JM \& M Moran, 2008, Increasing marginal returns and the danger of collapse of commercially valuable fish stocks, Ecol Econ 68, 422428.

Messick DM \& MB Brewer, 1983, Solving social dilemmas: a review, Annual Rev Personality 83 Social Psychology 4, 11-43.

Messick DM, H Wilke, MB Brewer, PM Kramer, PE Zemke \& L Lui, 1983, Individual adaptation and structural change as solutions to social dilemmas, J Personality \& Social Psychology 44, 294-309.

Olson M, 1965, "The Logic of Collective Action", Harvard University Press, Cambridge, MA.

Ophuls W, 1973, Leviathan or oblivion, in: "Toward a Steady State Economy" (HE Daly, ed.), Freeman, San Francisco, pp. 215-230.

Ostrom E, 1990, "Governing the Commons: The Evolution of Institutions", Cambrige University Press, London, UK.

Ostrom E, 2005, "Understanding Institutional Diversity", Princeton University Press, Princeton, NJ.

Ostrom E, R Gardner \& J Walker, 1994, "Rules, Games, and CommonPool Resources", The University of Michigan Press, Ann Arbor, MI.

Phelps ES \& RA Pollack, 1968, On second-best national saving and game equilibrium growth, Rev Econ Studies 35, 185-199.

Platt J, 1973, Social traps, Amer Psychologists 28, 641-651.

Poundstone W, 1992, "Prisoner's Dilemma", $1^{\text {st }}$ Anchor Books Edition, NY.

Rabl A, 1996, Discounting of long-term costs: what would future generations prefer us to do? Ecol Econ 17, 137-145.

Rachlin H, 1970 "Introduction to Modern Behavioralism", Freeman, San Francisco.

Rachlin H \& L Green, 1972, Commitment, choice and self-control, $J$ Experimental Animal Behavior 48, 347-355.

Rousseau JJ, 1755, Discours sur l'Origine et les Fondemens de l'Inégalité parmi les Hommes (Original spelling), Amsterdam.

Rubinstein A, 2003, Is it 'economics and psychology'? The case of hyperbolic discounting, Intern Econ Rev 44, 1207-1216.

Samuelson P, 1937, A note on measurement of utility, Rev Econ Studies 4, 155-161.

Schelling TC, 1978, "Micromotives and Macrobehavior", WW Norton, NY.

Schelling TC, 1984, "Choice and Consequence: Perspectives of an Errant Economist", Harvard University Press, Cambridge, MA.

Schelling TC, 1995, Intergenerational discounting, Energy Policy 23, 395401.

Sen A, 1967, Isolation, assurance and the social rate of discount, Quart $J$ Econ 81, 112-124. 
Sigmund K, 2010, "The Calculus of Selfishness", Princeton University Press, Princeton, NJ.

Skyrms B, 1996, "Evolution of the Social Contract", Cambridge University Press, Cambridge, UK.

Skyrms B, 2001, The Stag Hunt, Proc $\mathcal{E}$ Addr Amer Philosophical Assoc 75, 31-41.

Skyrms B, 2004, "The Stag Hunt and the Evolution of Social Structure", Cambridge University Press, Cambridge, UK.

Smith RJ, 1981, Resolving the Tragedy of the Commons by creating private property rights in wildlife, CATO $J \mathbf{1}, 439-468$.

Snyder GH \& P Diesing, 1977, "Conflict Among Nations: Bargaining, Decision Making, and System Structure in International Crises", Princeton University Press, Princeton, NJ.

Strotz R, 1956, Myopia and inconsistency in dynamic utility maximization, Rev Econ Studies 23, 165-180.

Tversky A \& D Kahneman, 1981, The framing of decisions and the psychology of choice, Science 211, 453-458.

Tversky A \& D Kahneman, 1986, Rational choice and the framing of decisions, J Business 59, 251-278.

Warner JT \& S Pleeter, 2001, The personal discount rate: Evidence from military downsizing programs, Am Econ Rev 91, 33-53.

Weitzman ML, 1998, Why the far-distant future should be discounted at its lowest possible rate, J Environmental Econ $\&$ Management 36, 201-208.

Weitzman ML, 2001, Gamma discounting, Amer Econ Rev 91, 260-271. 